Research Paper

\title{
Role of Serum Biomarkers in Early Detection of Diabetic Cardiomyopathy in the West Virginian Population
}

\author{
Adam Shaver ${ }^{*}$, Alexandra Nichols ${ }^{1 *}$, Ellen Thompson², Amrita Mallick ${ }^{1}$, Kristen Payne ${ }^{1}$, Chris Jones ${ }^{2}$, \\ Nandini D. P. K. Manne ${ }^{3}$, Shanmuga Sundaram ${ }^{1}$, Joseph I. Shapiro ${ }^{1}$, and Komal Sodhi ${ }^{4}$ \\ 1. Department of Medicine Joan C. Edwards School of Medicine, Marshall University; \\ 2. Department of Cardiology, Marshall University; \\ 3. Department of Public Health, Marshall University; \\ 4. Department of Surgery and Pharmacology, Marshall University, USA \\ * Both authors contributed equally \\ $\square$ Corresponding author: Komal Sodhi, M.D., Assistant Professor of Surgery and Pharmacology, Marshall University Joan C Edwards School of Medicine, WV \\ 25701, Tel: 304 691-1700, Fax: 914 347-4956, E-mail: Sodhi@marshall.edu.
}

(-) Ivyspring International Publisher. Reproduction is permitted for personal, noncommercial use, provided that the article is in whole, unmodified, and properly cited. See http://ivyspring.com/terms for terms and conditions.

Received: 2015.10.10; Accepted: 2016.01.04; Published: 2016.02.05

\begin{abstract}
Objectives: Diabetic cardiomyopathy (DCM) is an established complication of diabetes mellitus. In West Virginia, the especially high incidence of diabetes and heart failure validate the necessity of developing new strategies for earlier detection of DCM. Since most DCM patients remain asymptomatic until the later stages of the disease when the fibrotic complications become irreversible, we aimed to explore biomarkers that can identify early-stage DCM. Methods: The patients were grouped into 4 categories based on clinical diabetic and cardiac parameters: Control, Diabetes (DM), Diastolic dysfunction (DD), and Diabetes with diastolic dysfunction (DM+DD), the last group being the preclinical DCM group. Results: Echocardiography images indicated severe diastolic dysfunction in patients with DD+DM and DD compared to DM or control patients. In the $D M$ and $D M+D D$ groups, TNFa, isoprostane, and leptin were elevated compared to control $(p<0.05)$, as were clinical markers $\mathrm{HDL}$, glucose and hemoglobin AlC. Fibrotic markers IGFBP7 and TGF- $\beta$ followed the same trend. The Control group showed higher beneficial levels of adiponectin and bilirubin, which were reduced in the $D M$ and $D M+D D$ groups $(p<0.05)$. Conclusion: The results from our study support the clinical application of biomarkers in diagnosing early stage DCM, which will enable attenuation of disease progression prior to the onset of irreversible complications.
\end{abstract}

Key words: cardiomyopathy, diabetes, West Virginia, serum biomarkers.

\section{Introduction}

In recent years, diabetes mellitus (DM) has become a national health epidemic. The Centers for Disease Control and Prevention reports that West Virginia has one of the highest rates of diabetes in the United States with more than $11 \%$ of the population affected. The Framingham Heart Study revealed that the risk of heart failure is up to 5 times higher in diabetics than non-diabetics, when controlling for other risk factors. Diabetic Cardiomyopathy (DCM) is an established complication of diabetes [1-6] that involves abnormal relaxation of the ventricles, referred to as diastolic dysfunction, with concurrent hypertrophy of cardiomyocytes [6, 7]. Diastolic dysfunction is thought to be the first functional abnormality in DCM and can be seen in 40-60\% of asymptomatic diabetic patients through echocardiographic imagining studies $[2,6]$. Diabetic patients with subclinical diastolic dysfunction have a 5-year mortality rate of $30.8 \%$ 
compared to $12.1 \%$ for diabetic patients with no diastolic dysfunction [4]. As DCM enters its later stage, it progresses from diastolic dysfunction to overt stage $\mathrm{C}$ heart failure with preserved ejection fraction, which has no proven effective treatment [7], thus validating the importance of identifying biomarkers that can improve detection of DCM prior to the onset of irreversible complications.

Diabetes impairs glucose uptake and results in an increase in fatty acid (FA) metabolism in cardiac tissue $[3,8,9]$. In diabetes, decreased insulin signaling activates transcriptional signaling pathways that induce the expression of genes involved in stimulating FA uptake; however the uptake of FAs exceeds metabolic demand and results in triglyceride and cholesterol accumulation in the myocardium which impairs diastolic function [8-11]. A study by McGavock et al compared normoglycemic individuals with diabetic patients and confirmed a positive correlation between impaired glucose tolerance and myocardial triglyceride content and found that triglyceride accumulation preceded the onset of ventricular dysfunction [11]. Abnormal FA metabolism also leads to depressed levels of high-density lipoprotein (HDL) [3].

Multiple studies have established a link between damage induced by oxidative stress and DCM [12, 13]. Damage from oxidative stress due to the chronic mitochondrial overproduction of reactive oxygen species (ROS) plays a crucial role in inflammation and results in irreversible fibrosis and cardiomyocyte death $[2,12,14,15]$. Inflammation in the myocardium is mediated by pro-inflammatory cytokines including TNFa and interleukin-6 [16]. Isoprostanes are formed by the peroxidation of polyunsaturated FAs and are considered an accurate reflection of the extent of oxidative damage [17]. Amelioration of oxidative stress on a molecular level can be achieved through induction of antioxidant agents, and studies have shown that enhancing mitochondrial ROS scavenging systems mitigates diabetes-induced cardiac dysfunction $[2,12,18-21]$. Bilirubin, a product of heme catabolism, is a potent antioxidant and under normal physiological conditions may attenuate many ROS-derived complications of DCM [22, 23]. Adiponectin is a hormone secreted by adipose tissue that regulates metabolic processes and functions as an antioxidant; the low plasma levels of adiponectin seen in diabetes contribute to the oxidative damage seen in DCM [24, 25].

Structurally, the progression of DCM has been linked to cardiomyocyte hypertrophy and increased fibrosis [26-29]. The presence of cardiomyocyte hypertrophy was supported by data from The Framingham Heart Study, which revealed left ventricular mass was higher in diabetics compared to non-diabetics independent of covariates [30]. Hyperglycemia facilitates the reaction of glucose with collagen to form advanced glycation end-products (AGEs) that promote the crosslinking of collagen molecules to produce fibrosis [26]. Insulin-like growth factor binding protein 7 (IGFBP7) is a modulator of insulin-like growth factors, which actively regulate insulin consumption and receptor activity [27]. IGFBP7 has been identified as a biomarker for diastolic dysfunction associated with cardiac hypertrophy, myocyte fibrosis, and vascular remodeling, and an association exists between serum IGFBP7 levels and echocardiographic abnormalities describing diastolic heart dysfunction independent of systolic function [28]. In diabetics, increased ROS production causes the subsequent release of the profibrotic marker transforming growth factor beta (TGF- $\beta$ ) [29]. TGF- $\beta$ levels correlate with the degree of cardiac fibrosis [26]. The reliable early detection of fibrotic changes in DCM patients by IGFBP7 and TGF- $\beta$ is clinically pertinent since current experimental therapies designed to prevent progression of DCM include targeting collagen deposition and fibrosis through AGE inhibitors and AGE cross-link breakers $[1,6]$.

The aim of this study was to investigate a panel of biomarkers, including fibrotic markers, antioxidants, markers of oxidative stress and a metabolic profile that can be used to detect DCM prior to the presentation of clinical signs. Early stage DCM is initially asymptomatic and reversible; however, DCM is a progressive disease and at the later stage prognosis becomes poor. Analysis of biomarkers would be a simple, noninvasive diagnostic tool with a high predictive value for clinical use in patients with the potential to develop DCM, which would allow for the implementation of therapeutic strategies to be started in time to delay and mitigate the disease progression.

\section{Materials and Methods}

\section{Patients}

This study comprised of 100 patients, divided into 4 categories based on predetermined clinical diabetic and cardiac parameters: 1. Control; 2. Diabetes with normal diastolic function; 3. Diastolic dysfunction without diabetes; 4 . Diabetes and diastolic dysfunction. Contraindications to inclusion included any systolic dysfunction observed through echocardiography, evidence of prior myocardial infarction, known valvular or congenital heart disease or other significant comorbidities, including malignancy, renal failure, or significant psychiatric illness. The study was approved by the Ethics Committee of Cabell Huntington Hospital, West Virginia. All of the subjects signed a written informed consent form. 
Table 1: Demographic Profile

\begin{tabular}{lllll}
\hline Groups & Control & $\begin{array}{l}\text { Diastolic } \\
\text { dysfunction } \\
\text { (DD) }\end{array}$ & $\begin{array}{l}\text { Diabetes } \\
\text { mellitus } \\
\text { (DM) }\end{array}$ & $\begin{array}{l}\text { Diabetes mellitus with } \\
\text { diastolic dysfunction } \\
\text { (DM+DD) }\end{array}$ \\
\hline Age (yrs.) & $41.67 \pm 11.79$ & $44.27 \pm 8.31$ & $48 \pm 11.78$ & $45.69 \pm 8.42$ \\
Sex (M/F) & $43 \% / 57 \%$ & $50 \% / 50 \%$ & $52 \% / 48 \%$ & $57 \% / 43 \%$ \\
\hline
\end{tabular}

\section{Blood Samples}

Venous blood was drawn from an antecubital vein in heparinized and Vacutainer tubes for serum levels of TNFa, IL-6, Isoprostane, leptin, adiponectin, bilirubin, IGFBP7, TGF- $\beta$ and lipid and glucose profiles. Blood was processed after collection and serum collected for analysis. Serum was collected by spinning down the vacutainer tube at $2000 \mathrm{rpm}$ for 5 minutes at $4^{\circ} \mathrm{C}$ and stored at $-80^{\circ} \mathrm{C}$ until analysis.

\section{Analysis of Biomarkers}

Serum samples frozen at $-80^{\circ} \mathrm{C}$ were analyzed for levels of the following biomarkers through enzyme-linked immunosorbent-assay (ELISA): TNFa, IL-6, adiponectin and leptin (EMD Millipore, Temecula, Ca), Isoprostane (Cayman Chemical Company, Ann Arbor, MI), bilirubin (Sigma-Aldrich, St. Louis, $\mathrm{MO}$ ), triglycerides (Abcam, Cambridge, MA), and IGFBP7 and TGF- $\beta$ (kits from R\&D Systems, Minneapolis, MN). ELISAs were conducted in accordance to the manufacturer's protocol.

\section{Echocardiography}

Patients underwent echocardiography with 2D, Doppler, and color flow imaging within the year prior to enrollment. Studies were performed on a Philips IE 33 with an S5 transducer by one of 2 certified echo technicians in an ICAEL- accredited laboratory. Measurements were obtained in accordance with guidelines set forth by the American Society of Echocardiography [31] 2D imaging was used to assess ejection fraction (EF), septal wall thickness, posterior wall thickness and left atrial size. Relative wall thickness was calculated with posterior and septal wall thickness by $(2 \times$ PWTd)/LVIDd [32]. Mitral valve in- flow Doppler was recorded, including E and A waves. Tissue Doppler was used for $\mathrm{E}^{\prime}$ and $\mathrm{A}^{\prime}$. Echocardiographic images were read by physicians who were blinded to the rest of the study.

\section{Statistical Analysis}

Data were analyzed using Graphpad Prism 4.0. Equal variance was assured by Bartlett's test for each biomarker within each of the four patient categories. ANOVA was conducted to identify statistically significant differences in the mean serum levels for the different biomarkers. The Tukey post-hoc test was used to indicate which patient groups showed statistically significant differences for the biomarker level measured.

\section{Results}

\section{Echocardiography}

All patients underwent transthoracic echocardiography to evaluate left ventricular diastolic filling pressure. Patients were identified as normal or as having diastolic dysfunction (DD), with DD encompassing a spectrum of severities including impaired relaxation, pseudonormal and restrictive diastolic dysfunction (Fig. 1). Results from echocardiographic imaging studies were used in conjunction with the diabetic status of the patients to establish the 4 patient categories.

\section{TNFa, isoprostane and IL-6 levels}

TNFa, isoprostanes, and IL-6 are inflammatory mediators that correlate with the extent of oxidative damage in tissues. TNFa and IL-6 were found to be elevated in DM, DD and DM+DD patients when compared to the control group (Fig. 2A and B respectively; $\mathrm{p}<0.05$ ). Levels of TNFa and IL-6 were significantly higher in patients with DM+DD than the levels found in either the patients with DM or the patients with DD alone $(p<0.05)$. Patients with DM and patients with DM+DD had increased levels of isoprostane compared to the control (Fig. 2C). Additionally, isoprostane levels in patients with $\mathrm{DM}+\mathrm{DD}$ were higher than levels observed in patients with DD alone $(\mathrm{p}<0.05)$.

Table 2: Patient Clinical Profile.

\begin{tabular}{lllll}
\hline & BMI & Triglycerides $(\mathrm{mg} / \mathrm{dL})$ & HDL $(\mathrm{mg} / \mathrm{dL})$ & Glucose $(\mathrm{mg} / \mathrm{dL})$ \\
\hline Control & $23.89( \pm 1.01)$ & $61.12( \pm 11.02)$ & $67.60( \pm 3.96)$ & $91.50( \pm 4.50)$ \\
DM & $39.79^{*}( \pm 1.82)$ & $85.72( \pm 8.62)$ & $43.60^{*}( \pm 2.04)$ & $186.28^{*}( \pm 3.97)$ \\
DD & $31.42 \#( \pm 2.70)$ & $92.16( \pm 8.10)$ & $44.42^{*}( \pm 3.85)$ & $107.23 \#( \pm 24.14)$ \\
DM+DD & $39.44^{*}( \pm 2.68)$ & $113.05^{*}( \pm 9.39)$ & $39.50^{*}( \pm 1.97)$ & $5.04^{*}( \pm 0.12)$ \\
\hline
\end{tabular}

Values represent means \pm SEM. * $p<0.05$ vs Control; $\# p<0.05$ vs DM; $+p<0.05$ vs DD. 

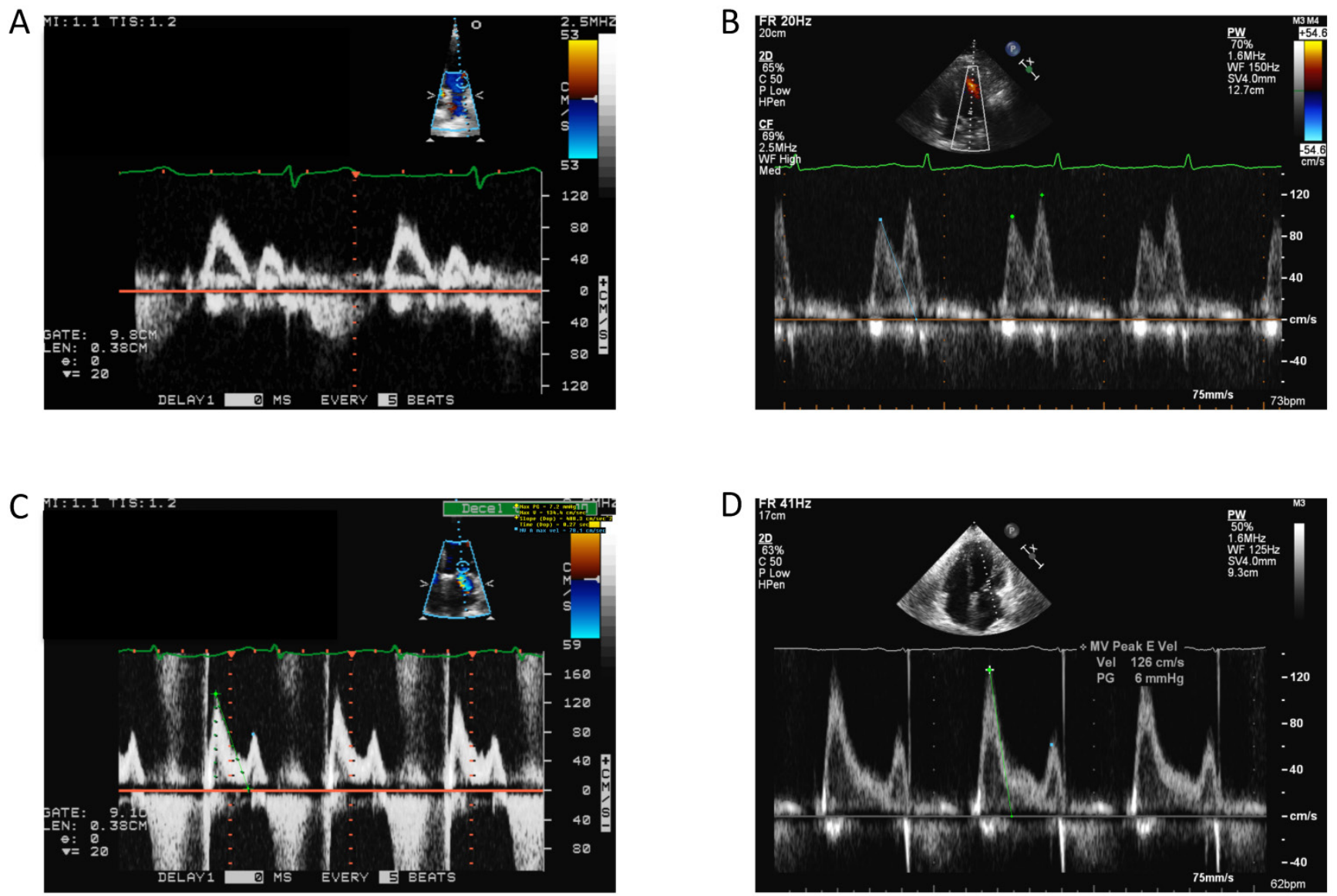

Figure 1: Mitral inflow velocities representing normal physiology as well as the spectrum of diastolic dysfunction. A: Normal. B: Impaired Relaxation. C: Grade II diastolic dysfunction (pseudonormalization). D: Restrictive

A

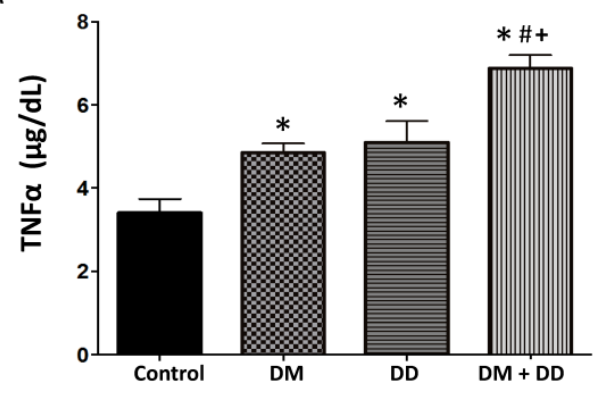

B

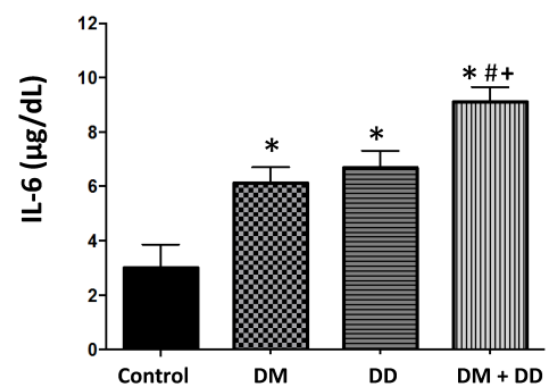

C

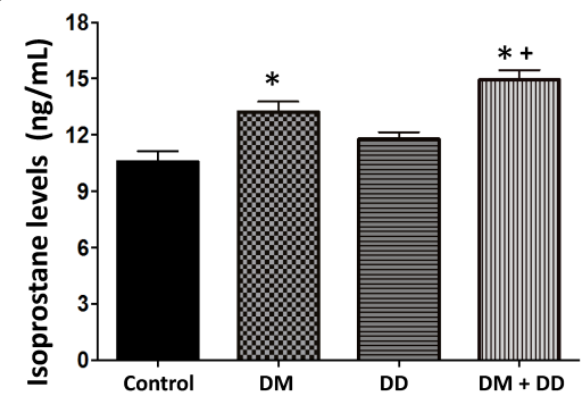

Figure 2: Assay of serum concentrations of (A) TNFa, (B) IL-6 and (C) Isoprostane in Control, DM, DD and DM+DD patient groups. Values represent means \pm SEM. $* p<0.05$ vs CTR; $\# p<0.05$ vs DM, $+p<0.05$ vs DD. 


\section{Leptin, adiponectin and bilirubin levels}

Leptin is an adipose-derived hormone that stimulates energy expenditure and is proportional to the amount of body fat. Leptin was significantly higher in the DM, DD and DM+DD patients when compared to the control group and was also significantly increased in the DM+DD patients when compared to the patients with DD alone (Fig. 3A; $p<0.05$ ). Adiponectin was significantly lower in DM, DD, and $\mathrm{DM}+\mathrm{DD}$ patients when compared to control groups (Fig.3B). Adiponectin was also significantly decreased in $\mathrm{DM}+\mathrm{DD}$ patients when compared to patients with DM and DD alone $(p<0.05)$. Bilirubin, a potent anti-

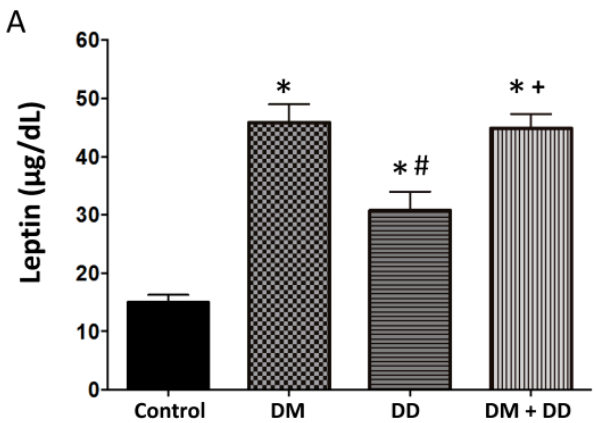

oxidant, was found to be lowered in the DM+DD group compared to the control (Fig. 3C; $\mathrm{p}<0.05$ ).

\section{TGF- $\beta$ and IGFBP7 levels}

TGF- $\beta$ correlates to the extent of fibrosis and IGFBP7 has been linked to cardiomyocyte hypertrophy and fibrosis. In concordance with our hypothesis, TGF- $\beta$ levels were elevated in DM, DD and DM+DD patients when compared to the control group (Fig. 4A; $\mathrm{p}<0.05)$. DM+DD patients did have significantly higher TGF- $\beta$ levels than the DM group alone. IGFBP7 was found to be increased in the DM, DD and $\mathrm{DM}+\mathrm{DD}$ groups compared to the control. IGFBP7 was also higher in patients with DM+DD compared to patients with DM or DD alone (Fig. 4B; $\mathrm{p}<0.05$ ).

B

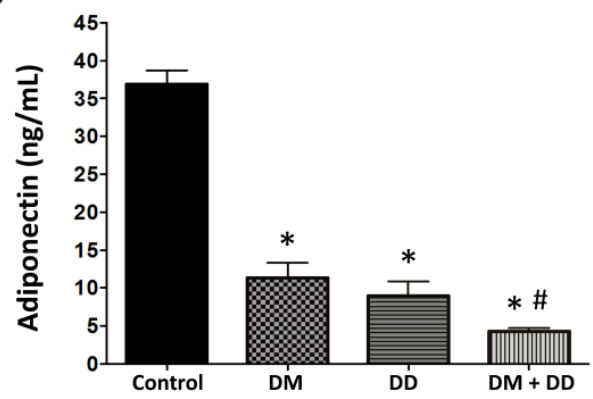

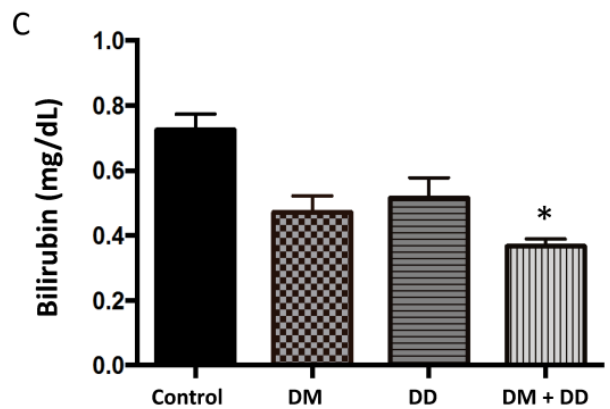

Figure 3: Serum levels of (A) Leptin, (B) Adiponectin and (C) Bilirubin in Control, DM, DD and DM+DD patient groups determined by ELISA. Values represent means \pm SEM. $* p<0.05$ vs CTR; $\# p<0.05$ vs DM, $+p<0.05$ vs DD.

A

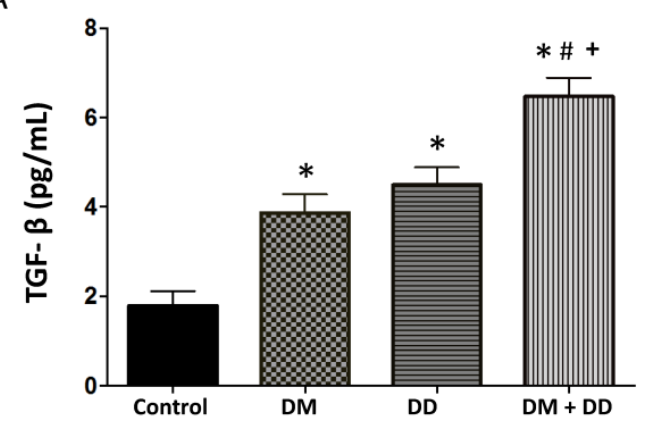

B

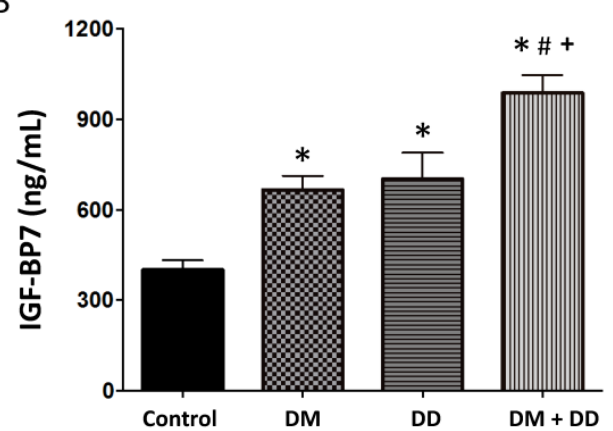

Figure 4: Assay of serum concentrations of (A) TGF- $\beta$ and (B) IGFBP7 in Control, DM, DD and DM+DD patient groups. Values represent means \pm SEM. * $p<0.05$ vs CTR; $\# p<0.05$ vs DM, $+p<0.05$ vs DD. 


\section{Discussion}

This study demonstrated that a panel of biomarkers in conjunction with echocardiography plays an important role in the detection of DCM prior to the manifestation of symptoms and irreversible complications. Our data indicated that early stage DCM can be characterized by elevated levels of inflammatory markers, decreased antioxidant markers and, most importantly, increased levels of the fibrotic markers IGFBP7 and TGF- $\beta$. These results, which are summarized in the schematic diagram (Fig. 5), identified potential biomarkers that can be used clinically to improve early diagnosis of DCM.

The clinical parameters studied include BMI and levels of triglycerides, HDL, glucose and HbA1C. A comprehensive national study, which substantiates the correlation between BMI and diabetes, found that individuals with diabetes were more likely to have a high BMI [33]. In concordance with established studies, our results demonstrated elevated blood glucose levels and $\mathrm{HbA} 1 \mathrm{C}$ levels in both $\mathrm{DM}$ patients and patients with DM+DD. As expected, neither glucose nor $\mathrm{HbA1C}$ was elevated in DD patients, because DD is not specific to DCM but can result from hypertension, aortic stenosis, myocardial infarction, or systolic heart failure [31]. Triglycerides are elevated in diabetic patients due to an increased reliance on fatty acid metabolism, and our data demonstrated DM+DD patients had significantly elevated triglyceride levels. Additionally, our data supported the accepted trend that diabetes is closely associated with reduced levels of HDL.

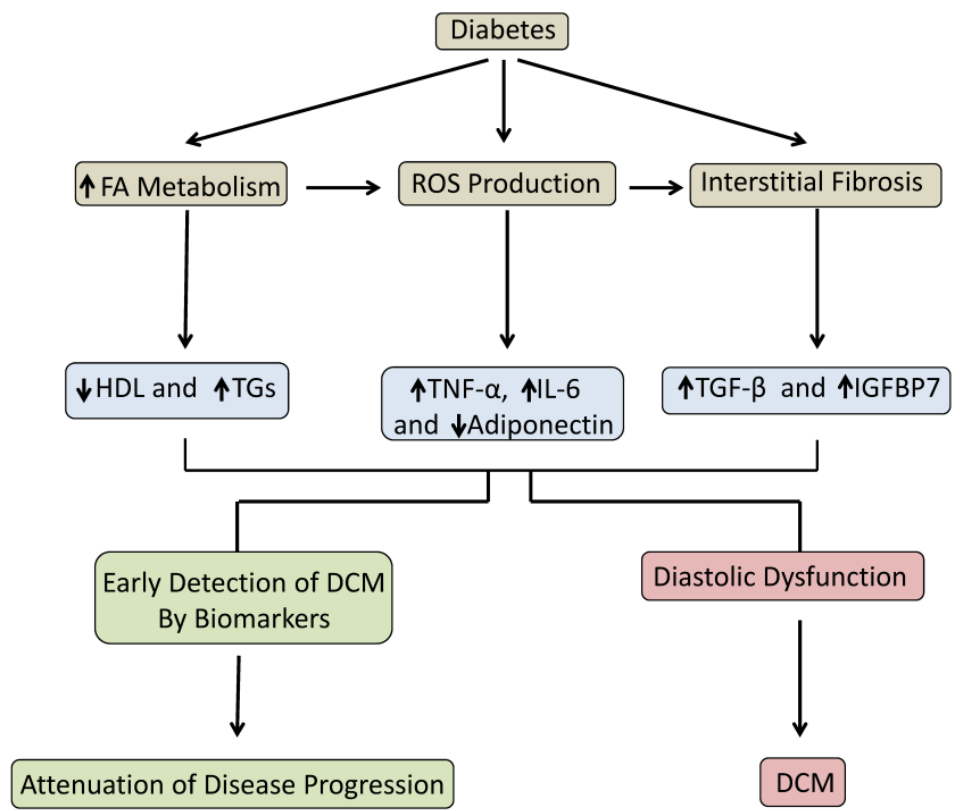

Figure 5: Schematic depicting the progression from diabetes to DCM and the role of biomarkers to detect early stage DCM and attenuate disease progression.
Elevated levels of the inflammatory cytokines TNFa and IL-6 are associated with diabetes, and previous studies have indicated their role in cardiac remodeling, fibrosis and cardiomyocyte apoptosis [34]. In corroboration with these findings, our study demonstrated elevated levels of TNFa and IL-6 relative to the control in DM, DD, and DM+DD patients, with the highest levels in DM+DD patients, supporting their utilization in the early detection of DCM and amelioration of disease progression. Isoprostane, an established marker of oxidative stress [11], was increased in patients with DM+DD. Our results also demonstrate a decreased level of the antioxidant bilirubin in patients with DM+DD, which supports previous findings that bilirubin is negatively associated with diabetes and its complications.

Leptin is closely associated with obesity and its comorbidities [35], and was highest in patients with $\mathrm{DM}$ and patients with DM+DD, which were the groups with the highest BMIs. There is an inverse relationship between adiponectin and leptin levels, with low adiponectin levels indicating metabolic and cardiovascular disease [36]. Adiponectin has antidiabetic effects and antagonizes the progression of DCM by exhibiting potent anti-hypertrophic activity in cardiomyocytes and diminishing cardiac cell death by inhibiting inflammation [36-38]. Consistent with previous studies, our data identified decreased levels of adiponectin in DM patients and DD patients and significantly lower adiponectin levels in DM+DD patients, which indicated a diminished ability to offset the DCM complications and progression.

IGFBP7 is an established marker for diabetes, and is associated with the severity of diastolic dysfunction [28]. IGFBP7 also has been implicated in fibrogenesis in diabetic patients, and increasing concentrations correlate with increased collagen deposition, which, in cardiac tissue, may contribute to diastolic stiffness [39]. Diabetes-induced ROS generation up-regulates the expression of the profibrotic marker TGF- $\beta$, leading to increased extracellular matrix synthesis and fibrosis [26]. Increased collagen deposition and interstitial fibrosis are key pathogenic components of DCM that result in myocardial stiffness, which manifests as diastolic dysfunction [2]. In concordance with previous studies, our data indicated elevated IGFBP7 and TGF- $\beta$ levels in $\mathrm{DM}, \mathrm{DD}$ and DM+DD patients, with DM+DD patients displaying the highest values. Therefore, these markers play an important role in the early detection of DCM and cardiac fibrosis, enabling early intervention to attenuate disease progression. 


\section{Conclusion}

In conclusion, our study identified a panel of biomarkers that can complement echocardiographic imaging studies to detect the diabetes-induced changes in cardiac structure and function present at the early stage of DCM. The progression of DCM from subclinical diastolic dysfunction to overt heart failure, and the associated high morbidity and mortality in the later stage of the disease, validates the need for reliable early diagnostic tools. The results of our clinical study can improve early diagnosis of DCM and help successfully implement preventative strategies that may slow or prevent development of the disease.

Our study advances the understanding of the clinical use of biomarkers in the diagnosis of DCM in a rural Appalachian population. Diabetes is a chronic disease with high prevalence in West Virginia, and its comorbidities present a significant public health concern. Therefore, West Virginia patients present the ideal population to study strategies for earlier detection of DCM. Positive results from this study will benefit this underserved population by enabling an earlier and more reliable diagnosis of DCM so that therapy can be administered in time to attenuate disease progression.

\section{Acknowledgement}

This work was supported by the National Institutes of Health Grants HL 109015, HL 071556 and HL 105649 (J.I.S.).

\section{Competing Interests}

The authors have declared that no competing interest exists.

\section{References}

1. Aneja A, Tang WH, Bansilal S, Garcia MJ, Farkouh ME. Diabetic cardiomyopathy: insights into pathogenesis, diagnostic challenges, and therapeutic options. The American journal of medicine. 2008; 121: 748-57. doi:10.1016/j.amjmed.2008.03.046.

2. Boudina S, Abel ED. Diabetic cardiomyopathy revisited. Circulation. 2007; 115: 3213-23. doi:10.1161/CIRCULATIONAHA.106.679597.

3. Diamant $\mathrm{M}$, Lamb HJ, Groeneveld $\mathrm{Y}$, Endert EL, Smit JW, Bax JJ, et al. Diastolic dysfunction is associated with altered myocardial metabolism in asymptomatic normotensive patients with well-controlled type 2 diabetes mellitus. Journal of the American College of Cardiology. 2003; 42: 328-35.

4. From AM, Scott CG, Chen HH. The development of heart failure in patients with diabetes mellitus and pre-clinical diastolic dysfunction a population-based study. Journal of the American College of Cardiology. 2010; 55: 300-5. doi:10.1016/j.jacc.2009.12.003.

5. Jagasia D, McNulty PH. Diabetes mellitus and heart failure. Congestive heart failure. 2003; 9: 133-9; quiz 40-1.

6. Miki $\mathrm{T}$, Yuda $\mathrm{S}$, Kouzu $\mathrm{H}$, Miura $\mathrm{T}$. Diabetic cardiomyopathy: pathophysiology and clinical features. Heart failure reviews. 2013; 18: 149-66. doi:10.1007/s10741-012-9313-3.

7. Lam CS. Diabetic cardiomyopathy: An expression of stage B heart failure with preserved ejection fraction. Diabetes \& vascular disease research. 2015. doi:10.1177/1479164115579006.

8. Stanley WC, Lopaschuk GD, McCormack JG. Regulation of energy substrate metabolism in the diabetic heart. Cardiovascular research. 1997; 34: 25-33.

9. Carley AN, Severson DL. Fatty acid metabolism is enhanced in type 2 diabetic hearts. Biochimica et biophysica acta. 2005; 1734: 112-26. doi:10.1016/j.bbalip.2005.03.005.
10. Sharma S, Adrogue JV, Golfman L, Uray I, Lemm J, Youker K, et al. Intramyocardial lipid accumulation in the failing human heart resembles the lipotoxic rat heart. FASEB journal : official publication of the Federation of American Societies for Experimental Biology. 2004; 18: 1692-700. doi:10.1096/fj.04-2263com.

11. McGavock JM, Lingvay I, Zib I, Tillery T, Salas N, Unger R, et al. Cardiac steatosis in diabetes mellitus: a $1 \mathrm{H}$-magnetic resonance spectroscopy study. Circulation. 2007; 116: 1170-5. doi:10.1161/CIRCULATIONAHA.106.645614.

12. Cai L, Wang Y, Zhou G, Chen T, Song Y, Li X, et al. Attenuation by metallothionein of early cardiac cell death via suppression of mitochondrial oxidative stress results in a prevention of diabetic cardiomyopathy. Journal of the American College of Cardiology. 2006; 48: 1688-97. doi:10.1016/j.jacc.2006.07.022.

13. Cai L. Suppression of nitrative damage by metallothionein in diabetic heart contributes to the prevention of cardiomyopathy. Free radical biology \& medicine. 2006; 41: 851-61. doi:10.1016/j.freeradbiomed.2006.06.007.

14. Frustaci A, Kajstura J, Chimenti C, Jakoniuk I, Leri A, Maseri A, et al. Myocardial cell death in human diabetes. Circulation research. 2000; 87: 1123-32.

15. Jay D, Hitomi H, Griendling KK. Oxidative stress and diabetic cardiovascular complications. Free radical biology \& medicine. 2006; 40: 183-92. doi:10.1016/j.freeradbiomed.2005.06.018.

16. Haugen E, Chen J, Wikstrom J, Gronros J, Gan LM, Fu LX. Parallel gene expressions of IL-6 and BNP during cardiac hypertrophy complicated with diastolic dysfunction in spontaneously hypertensive rats. International journal of cardiology. 2007; 115: 24-8. doi:10.1016/j.ijcard.2006.01.031.

17. Liu T, Stern A, Roberts LJ, Morrow JD. The isoprostanes: novel prostaglandin-like products of the free radical-catalyzed peroxidation of arachidonic acid. Journal of biomedical science. 1999; 6: 226-35. doi:25391.

18. Liang Q, Carlson EC, Donthi RV, Kralik PM, Shen X, Epstein PN. Overexpression of metallothionein reduces diabetic cardiomyopathy. Diabetes. 2002; 51: 174-81.

19. Matsushima S, Kinugawa S, Ide T, Matsusaka H, Inoue N, Ohta $Y$, et al. Overexpression of glutathione peroxidase attenuates myocardial remodeling and preserves diastolic function in diabetic heart. American journal of physiology Heart and circulatory physiology. 2006; 291: H2237-45. doi:10.1152/ajpheart.00427.2006.

20. Ye G, Metreveli NS, Donthi RV, Xia S, Xu M, Carlson EC, et al. Catalase protects cardiomyocyte function in models of type 1 and type 2 diabetes. Diabetes. 2004; 53: 1336-43.

21. Shen X, Zheng S, Metreveli NS, Epstein PN. Protection of cardiac mitochondria by overexpression of MnSOD reduces diabetic cardiomyopathy. Diabetes. 2006; 55: 798-805.

22. Zhao Y, Zhang L, Qiao Y, Zhou X, Wu G, Wang L, et al. Heme oxygenase-1 prevents cardiac dysfunction in streptozotocin-diabetic mice by reducing inflammation, oxidative stress, apoptosis and enhancing autophagy. PloS one. 2013; 8: e75927. doi:10.1371/journal.pone.0075927.

23. Abraham NG, Kappas A. Pharmacological and clinical aspects of heme $\begin{array}{lllll}\text { oxygenase. Pharmacological reviews. 2008; 60: } & \text { 79-127. }\end{array}$ doi:10.1124/pr.107.07104.

24. $\mathrm{Li} \mathrm{J}, \mathrm{Su} \mathrm{S}$, Zong X. Analysis of the association between adiponectin, adiponectin receptor 1 and diabetic cardiomyopathy. Experimental and therapeutic medicine. 2014; 7: 1023-7. doi:10.3892/etm.2014.1539.

25. Issan Y, Kornowski R, Aravot D, Shainberg A, Laniado-Schwartzman M, Sodhi $\mathrm{K}$, et al. Heme oxygenase-1 induction improves cardiac function following myocardial ischemia by reducing oxidative stress. PloS one. 2014; 9: e92246. doi:10.1371/journal.pone.0092246.

26. Asbun J, Villarreal FJ. The pathogenesis of myocardial fibrosis in the setting of diabetic cardiomyopathy. Journal of the American College of Cardiology. 2006; 47: 693-700. doi:10.1016/j.jacc.2005.09.050.

27. Ruan $\mathrm{W}$, Lai M. Insulin-like growth factor binding protein: a possible marker for the metabolic syndrome? Acta diabetologica. 2010; 47: 5-14. doi:10.1007/s00592-009-0142-3.

28. Gandhi PU, Gaggin HK, Sheftel AD, Belcher AM, Weiner RB, Baggish AL, et al. Prognostic usefulness of insulin-like growth factor-binding protein 7 in heart failure with reduced ejection fraction: a novel biomarker of myocardial diastolic function? The American journal of cardiology. 2014; 114: 1543-9. doi:10.1016/j.amjcard.2014.08.018.

29. Iglesias-De La Cruz MC, Ruiz-Torres P, Alcami J Diez-Marques L Ortega-Velazquez R, Chen S, et al. Hydrogen peroxide increases extracellular matrix mRNA through TGF-beta in human mesangial cells. Kidney international. 2001; 59: 87-95. doi:10.1046/j.1523-1755.2001.00469.x.

30. Palmieri V, Bella JN, Arnett DK, Liu JE, Oberman A, Schuck MY, et al. Effect of type 2 diabetes mellitus on left ventricular geometry and systolic function in hypertensive subjects: Hypertension Genetic Epidemiology Network (HyperGEN) study. Circulation. 2001; 103: 102-7.

31. Nagueh SF, Appleton CP, Gillebert TC, Marino PN, Oh JK, Smiseth OA, et al. Recommendations for the evaluation of left ventricular diastolic function by echocardiography. Journal of the American Society of Echocardiography : official publication of the American Society of Echocardiography. 2009; 22: 107-33. doi:10.1016/j.echo.2008.11.023.

32. Lang RM, Bierig M, Devereux RB, Flachskampf FA, Foster E, Pellikka PA, et al. Recommendations for chamber quantification. European journal of echocardiography : the journal of the Working Group on Echocardiography of 
the European Society of Cardiology. 2006; 7: 79-108. doi:10.1016/j.euje.2005.12.014.

33. Bays HE, Chapman RH, Grandy S, Group SI. The relationship of body mass index to diabetes mellitus, hypertension and dyslipidaemia: comparison of data from two national surveys. International journal of clinical practice. 2007; 61: 737-47. doi:10.1111/j.1742-1241.2007.01336.x.

34. Sun M, Chen M, Dawood F, Zurawska U, Li JY, Parker T, et al. Tumor necrosis factor-alpha mediates cardiac remodeling and ventricular dysfunction after pressure overload state. Circulation. 2007; 115: 1398-407. doi:10.1161/CIRCULATIONAHA.106.643585.

35. Ruhl CE, Harris TB, Ding J, Goodpaster BH, Kanaya AM, Kritchevsky SB, et al. Body mass index and serum leptin concentration independently estimate percentage body fat in older adults. The American journal of clinical nutrition. 2007; 85: 1121-6.

36. Shibata R, Ouchi N, Ito M, Kihara S, Shiojima I, Pimentel DR, et al. Adiponectin-mediated modulation of hypertrophic signals in the heart. Nature medicine. 2004; 10: 1384-9. doi:10.1038/nm1137.

37. Holland WL, Miller RA, Wang ZV, Sun K, Barth BM, Bui $\mathrm{HH}$, et al Receptor-mediated activation of ceramidase activity initiates the pleiotropic actions of adiponectin. Nature medicine. 2011; 17: 55-63. doi:10.1038/nm.2277.

38. Battiprolu PK, Lopez-Crisosto C, Wang ZV, Nemchenko A, Lavandero S, Hill JA. Diabetic cardiomyopathy and metabolic remodeling of the heart. Life sciences. 2013; 92: 609-15. doi:10.1016/j.lfs.2012.10.011.

39. Guo XH, Liu LX, Zhang HY, Zhang OQ Li Y, Tian XX, et al. Insulin-like growth factor binding protein-related protein 1 contributes to hepatic fibrogenesis. Journal of digestive diseases. 2014; 15: 202-10. doi:10.1111/1751-2980.12126. 